\title{
Editorial
}

\section{Epigenetic alterations related to early life stressful events}

In recent years we have begun to understand how our environment influences us and shapes our lives down to the smallest molecular level. The different challenges we face at all stages in life shape our epigenetic landscape. Ventura-Junca et al. summarise and discuss this new fast evolving field in this issue of $A C N$. They highlight different epigenetic mechanisms that may become perturbed by stressful events early in life and which influence it may have on our physical and mental health. Even before we are born our epigenetic patterns are affected by the environment. Heijmans et al. reported in 2008 that prenatal exposure to famine during the Dutch Hunger Winter 1944-1945 is associated with lower DNA methylation of the imprinted $I G F 2$ gene six decades later (1). The hypomethylation may be related to a deficiency in methyl donors, such as the amino acid methionine. However, an additional contribution of other stressors, such as cold and emotional stress, cannot be ruled out. Thus prenatal famine may reflecting 'a very early life stressful event'.

How we enter the world may also affect our epigenome. The stress of being born exceeds that of any other critical life event. It has been suggested that the global DNA methylation pattern in leukocytes is higher if we are born by caesarean section (CS) compared to vaginal delivery (VD) (2). After 3-5 days no significant difference between CS and VD could be detected, but this does not exclude that local differences remain. Once born our upbringing continues to affect our epigenetics. It has been demonstrated in a rat model that how the parents groom their offspring influences the epigenetic pattern on specific genes in the brain (3). This has been supported by studies in abused/neglected monkeys and humans, reviewed in this issue of Ventura-Junca 2012. Even in our adult life, after the sensitive childhood and adolescence we continue to be shaped by our environment. Studies on monozygotic twins show bigger differences in DNA methylation with age $(4,5)$. The comparisons of monozygotic twins suggest that external and/or internal factors can have an impact in the phenotype by altering the pattern of epigenetic modifications throughout life.

Epigenetic events alter the chromatin structure and regulate gene expression. Perturbed epigenetic regulation has been associated with depression-related behaviour. The promising property of epigenetics contra genetics is that epigenetic modifications are reversible. They are created in a chemical equilibrium of, e.g. histone acetylases and histone deacetylases. This opens up a possibility to manipulate and reset the epigenetic landscape. In mouse visual cortex, this proof of principle has already been shown. Treatment with HDAC inhibitors reset the histone acetylation pattern in adult mice to a histone acetylation level that resembles the levels during the critical period when the mouse is young (6). The restored epigenetic landscape was suggested to induce a new critical period. This 'time travel' where we can make an adult visual cortex 'young' again by epigenetic manipulation opens up for novel potential possibilities to treat various conditions. One may speculate that an epigenetic modulation caused by early stressful events in life can be corrected in adulthood with specific epigenetic drugs. In this line, the antidepressant potential of HDAC inhibitors has been supported by experiments in animal models (7). Moreover, we have recently shown that administration of the antidepressant, escitalopram (a selective serotoninreuptake inhibitor) induces DNA demethylation in the P11 promoter and re-establishes a 'non pathological' DNA methylation pattern in a rat model (8). Many questions remain to be answered before we understand what consequences the modulated epigenetic landscape has on us.

Another problem with epigenetic treatment is the specificity of the epigenetic drugs available today. The HDAC inhibitors on the market often inhibit whole classes of HDACs, not specific ones. This causes unnecessary side-effects. To be able to tailor 


\section{Editorial}

the epigenetic treatment both better understanding of the regulating epigenetic mechanisms and more specific drugs are needed. However, treatment with DNA demethylating agents and unspecific HDAC inhibitors improves the survival of patients with specific subtypes of leukaemia $(9,10)$. This shows the vast potential of epigenetic therapy.

\section{Assistant Professor Andreas Lennartsson \\ Karolinska Institute, Stockholm, Sweden}

\section{References}

1. Heijmans BT, Tobi EW, Stein AD et al. Persistent epigenetic differences associated with prenatal exposure to famine in humans. Proc Natl Acad Sci U S A 2008;105: 17046-17049.

2. Schlinzig T, Johansson S, Gunnar A et al. Epigenetic modulation at birth - altered DNA-methylation in white blood cells after Caesarean section. Acta Paediatr 2009;98: 1096-1099.

3. Weaver IC, Cervoni N, Champagne FA et al. Epigenetic programming by maternal behavior. Nat Neurosci 2004;7: $847-854$.
4. Fraga MF, Ballestar E, Paz MF et al. Epigenetic differences arise during the lifetime of monozygotic twins. Proc Natl Acad Sci U S A 2005;102:10604-10609.

5. Talens RP, Christensen K, Putter H et al. Epigenetic variation during the adult lifespan: cross-sectional and longitudinal data on monozygotic twin pairs. Aging Cell 2012;11:694-703.

6. Silingardi D, Scali M, Belluomini G et al. Epigenetic treatments of adult rats promote recovery from visual acuity deficits induced by long-term monocular deprivation. Eur J Neurosci 2010;31:2185-2192.

7. Covington HE 3RD, Vialou VF, LaPlant $Q$ et al. Hippocampal-dependent antidepressant-like activity of histone deacetylase inhibition. Neurosci Lett 2011;493: 122-126.

8. Melas PA, Rogdaki M, Lennartsson A et al. Antidepressant treatment is associated with epigenetic alterations in the promoter of P11 in a genetic model of depression. Int J Neuropsychopharmacol 2012;15:669-679.

9. Keating GM. Azacitidine: a review of its use in the management of myelodysplastic syndromes/acute myeloid leukaemia. Drugs 2012;72:1111-1136.

10. Mercurio C, Minucci S, Pelicci PG. Histone deacetylases and epigenetic therapies of hematological malignancies. Pharmacol Res 2010;62:18-34. 